\title{
Secure closure of the tracheal incision after natural orifice transluminal endoscopic surgery with a silicone tracheal stent
}

\author{
Yun-Hen Liu, MD, Yi-Chen Wu, MD, Tzu-Ping Chen, MD, and Po-Jen Ko, MD, Tao-Yuan, Taiwan
}

Video clip is available online.

Since its introduction in 2004, natural orifice transluminal endoscopic surgery (NOTES) has attracted the interest of many clinicians and researchers and demonstrated technical feasibility and efficacy when used in various types of intra-abdominal operations, including transgastric liver biopsy, cholecystectomy, tubal ligation, and peritoneoscopy. ${ }^{1,2}$

Although NOTES has some potential advantages, such as being less invasive than the traditional method and causing less postoperative wound pain, there are certain challenges, including optimal access to the peritoneal and thoracic cavities, infection prevention, wound closure, and complication management. Among the abovementioned challenges,

\footnotetext{
From the Laboratory Animal Center, Department of Surgery, Chang-Gung Memorial Hospital at Linkou and Chang-Gung University, Tao-Yuan, Taiwan.

Supported by the Chang-Gung Memorial Hospital, Taiwan (contract no. CMRPG371471).

Disclosures: None.

Received for publication Oct 9, 2009; accepted for publication Nov 6, 2009; available ahead of print Feb 1, 2010.

Address for reprints: Po-Jen Ko, MD, Department of Thoracic and Cardiovascular Surgery, Chang Gung Memorial Hospital and Chang Gung University, No. 5, Fu-Shing Street, Guei-Shan Shiang, Tao-Yuan, Taiwan 333, China (E-mail: foreverairmail@ gmail.com).

J Thorac Cardiovasc Surg 2010;140:711-2

0022-5223/\$36.00

Copyright (c) 2010 by The American Association for Thoracic Surgery

doi:10.1016/j.jtcvs.2009.11.024
}

wound closure is probably the most difficult to achieve. Several endoscopic modalities, including endoclips, endosutures, and plicators, have been used for wound closure. However, none of these techniques is superior to the others. ${ }^{1-3}$

The bronchoscopic procedure is currently the most commonly used modality for treating central airway disease; this procedure has both diagnostic and therapeutic applications. Airway stenting has been used considerably in the treatment of patients with severe airway obstruction and has proved to be an efficient palliative treatment modality for tracheoesophageal fistula in patients with esophageal cancer. The aim of this study was to evaluate the feasibility of transtracheal examination of the pleural cavity and the effect of stenting for closing the tracheal incisions after NOTES.

\section{CLINICAL SUMMARY}

The Ethics Committee on Animal Research of the ChangGung Memorial Hospital approved the study protocol. In this preliminary study 2 canines ( 7 and $8 \mathrm{~kg}$, respectively) were used. After premedication (ketamine, $5 \mathrm{mg} \cdot \mathrm{kg}^{-1}$; xylazine $\mathrm{HC}, 10 \mathrm{mg} \cdot \mathrm{kg}^{-1}$ ), induction anesthesia was induced with isoflurane. Subsequently, the animals were orotracheally intubated. General anesthesia was maintained with isoflurane supplemented with intravenous infusion of xylazine HCL $\left(5 \mathrm{mg} \cdot \mathrm{kg}^{-1}\right)$.

First, a rigid bronchoscope was introduced into the lower trachea, and tracheal incisions were made at the lateral aspect of the right lower trachea $(3 \mathrm{~cm}$ above the carina) with a homemade metallic knife. The tracheal incision was subsequently dilated to $9 \mathrm{~mm}$ with a metallic tube. The

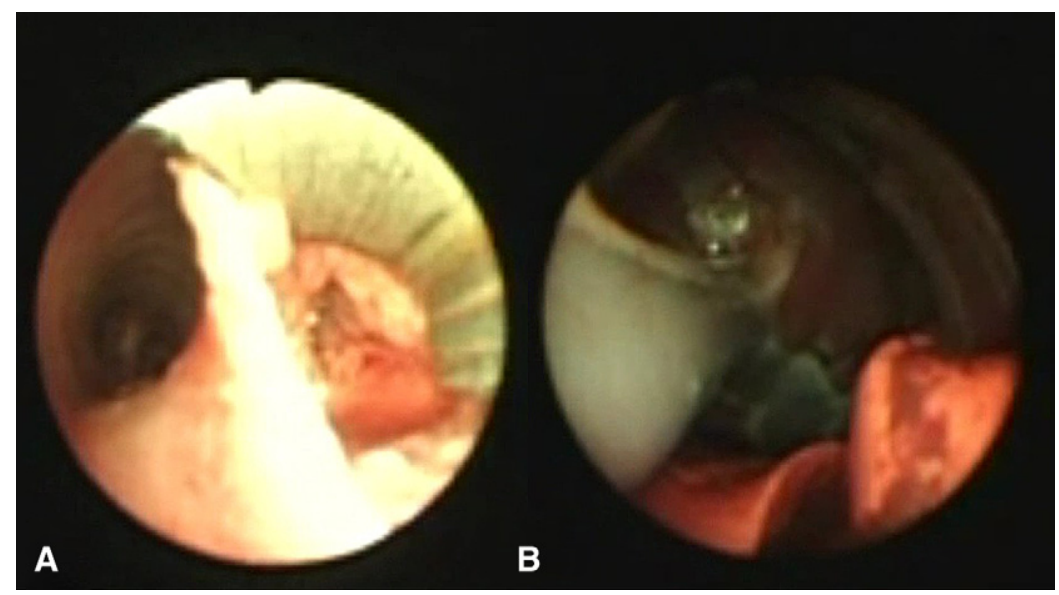

FIGURE 1. A, Transtracheal approach. B, Thoracic cavity exploration (inferior vena cava, lung, and diaphragm). 


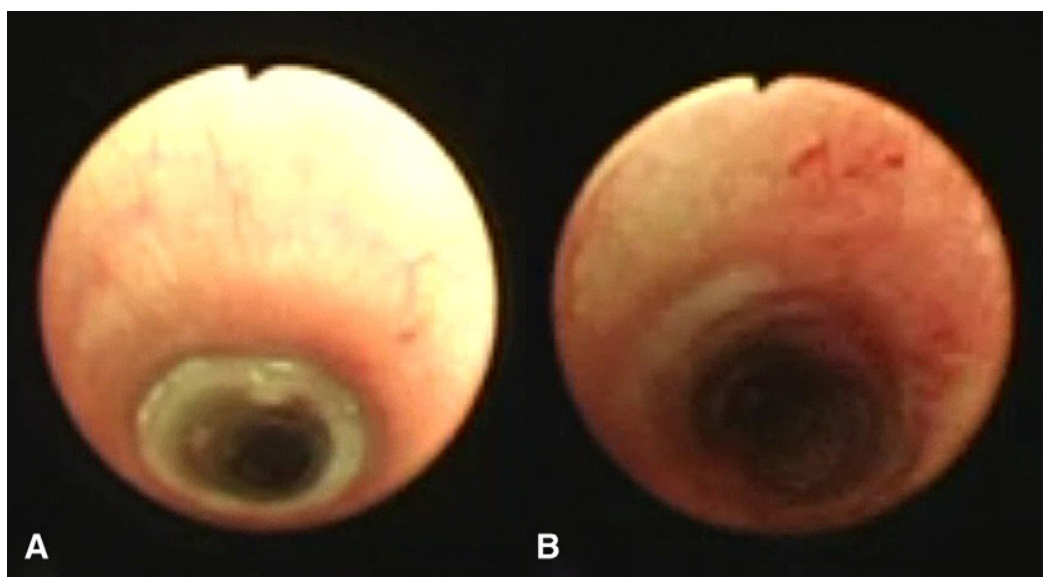

FIGURE 2. A, The tracheal wound was covered with a Dumon stent. B, Good healing of the tracheal wound 19 days after the operation.

thoracic cavity was explored through the tracheal opening through the metallic tube (Figure 1, A). The lung, mediastinum, diaphragm, and pleura were explored with a flexible bronchoscope (Figure 1,B). The tracheal incision was covered with a Dumon stent (Novatech, Grasse, France) after withdrawing the metallic tube from the pleural cavity (Figure 2, A). The animals were then allowed to recover from general anesthesia. The tracheal wound was evaluated by using bronchoscopy 2 weeks after the operation (Figure 2,B).

The operative times required for NOTES were 60 and 100 minutes, respectively. There were no major perioperative or postoperative complications, and all animals were alive at the end of the study period.

Both animals showed complete healing of the tracheal wound 2 weeks after the operation. Necropsy revealed that tracheal penetration had caused fibrosis in both dogs.

\section{DISCUSSION}

Tracheal injury poses a challenge to both patients and physicians. Major tracheal injury requires surgical intervention. For the treatment of minor tracheal injury or in patients who hesitate to undergo formal surgical reconstruction, conservative treatment is used, which is a relatively less invasive alternative to the surgical methods.

Advances in stent development and applications have improved the quality of life of patients with fistulas or rupture of major airways. The study by Choudhary and colleagues ${ }^{4}$ reported the successful palliation of tracheomediastinal fistulas after the placement of 3 different types of self-expanding metallic stents. Kim and associates ${ }^{5}$ also reported the feasibility of using a silicone-coated stent for the treatment of severe laceration of the left main bronchus. These findings suggest that endobronchial stenting for the treatment of airway rupture is not only safe but also minimizes the risk of further iatrogenic injury and can therefore be considered an alternative to conventional surgical airway reconstruction in patients with lesions that can be feasibly treated with stent placement.

Regarding the management of complications after placement of metallic stents to maintain airway patency, many authors have reported that airway fistulas that develop after metallic stent penetration can be successfully treated with silicone stent placement. On the basis of these reports, we recommend that only silicone stents should be used for tracheal wound closure in NOTES.

The data indicate that transtracheal examination of the thoracic cavity is a feasible and effective method in canine models. In our opinion it is difficult to develop an ideal entrance (tracheal wound) closure device. Nonetheless, the silicone stent is a feasible modality for entrance closure in NOTES because of the excellent treatment outcomes and because it can be easily adjusted and removed after treatment.

\section{References}

1. Sodergren MH, Clark J, Athanasiou T, Teare J, Yang GZ, Darzi A. Natural orifice translumenal endoscopic surgery: critical appraisal of applications in clinical practice. Surg Endosc. 2009;23:680-7.

2. Bergman S, Melvin WS. Natural orifice translumenal endoscopic surgery. Surg Clin North Am. 2008;88:1131-48.

3. Arezzo A, Morino M. Endoscopic closure of gastric access in perspective NOTES: an update on techniques and technologies. Surg Endosc. 2009 [Epub ahead of print].

4. Choudhary C, Gildea TR, Salman R, Guzman ED, Mehta AC. Management of tracheomediastinal fistula using self-expanding metallic stents. Ann Thorac Surg. 2008;85:1800-2.

5. Kim JH, Shin JH, Song HY, Lee HC, Kim KR, Park JH. Use of a retrievable metallic stent internally coated with silicone to treat airway obstruction. J Vasc Interv Radiol. 2008;19:1208-14. 